\title{
Polio eradication: a complex end game
}

\author{
Thomas Abraham examines the challenges of meeting the Global Polio Eradication Initiative's \\ target of eliminating polio by the end of this year
}

\section{Thomas Abraham director of public health communication programme}

Journalism and Media Studies Centre, University of Hong Kong, Hong Kong

Like the skipper of an ageing rust bucket trying to get to port before the boat goes under, the Global Polio Eradication Initiative is ploughing through choppy seas in its effort to stamp out polio and end a mission that began more than two decades ago. As the vessel lurches uncertainly on, a Greek chorus of commentators has raised questions about everything from the choice of vaccine and the technical strategies the campaign has used to the very wisdom of pursuing the goal of eradication.

These questions are important because they cast light on the long and tortuous route that the polio eradication programme has followed since 1988, when the World Health Assembly passed a resolution declaring it was committed to eradicating polio by 2000 . That deadline and a subsequent one have been missed, and a third deadline of the end of 2012 will probably be missed as well. Eradication seems both tantalisingly close and elusively distant. As it becomes harder to maintain the funding and government engagement necessary to keep the campaign going at the frenetic pace it has kept for several years now, there is a danger that the gains made over the past two decades will be lost, and the poliovirus, largely confined to pockets in South Asia and western and central Africa, will again entrench itself across swathes of the globe. $\Downarrow \Downarrow$

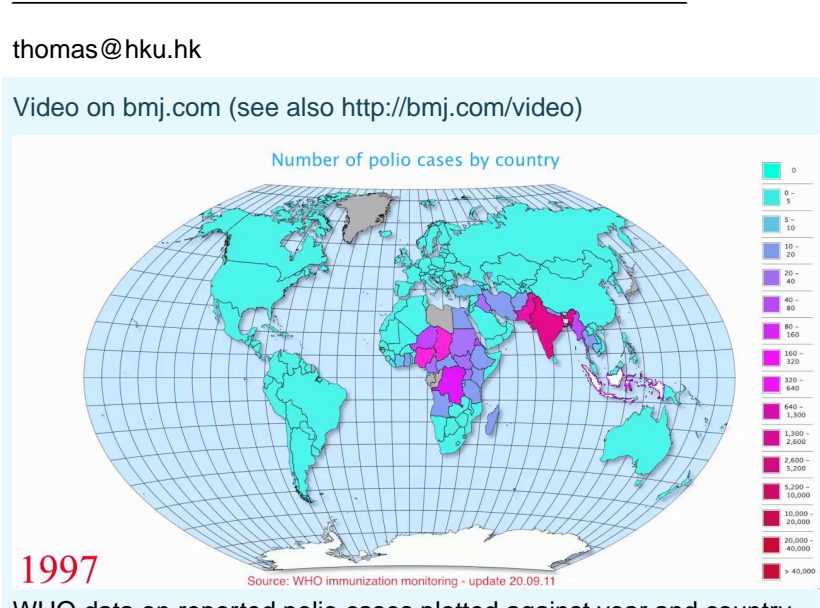

The initiative's key partners-WHO, Unicef, US Centers for Disease Control and Prevention, Rotary International, and the Bill and Melinda Gates Foundation-have responded by increasing pressure on governments in polio affected countries to intensify their immunisation and surveillance efforts. However, the response has been faltering because of difficulties such as underdeveloped health systems, poor governance, and chronic security problems.

On paper, the polio eradication campaign still aims to eradicate transmission of wild poliovirus by the end of 2012. But as the Independent Monitoring Board (IMB), a group of experts formed two years ago to evaluate and guide the programme, stated earlier this year, the campaign is "not on track to meet this goal." The board has suggested changes in strategy and execution to try to get back on track.

Both the polio campaign and the IMB have maintained that the world has to push ahead and complete eradication as soon as possible or face a resurgence of polio to the levels that existed before the campaign began. The IMB declared in its most recent report "The Global Polio Eradication Initiative has just two possible outcomes. Polio will be eradicated from the world, or it will not. The programme will succeed spectacularly, or fail monumentally." The IMB points out that it costs roughly \$1bn 
( $£ 628 \mathrm{~m} ; € 750 \mathrm{~m}$ ) a year to contain polio at its current low levels and argues that "if the eradication effort does not succeed soon, this funding will dry up." It warned that "failure would unleash the virus, paralyzing hundreds of thousands of children. This prospect seems unthinkable."

In response to these concerns, the World Health Assembly, the annual gathering of the WHO member countries, will discuss a resolution in May declaring polio a "programmatic emergency"- a move that will help focus global attention on polio eradication.

While polio campaigners have maintained a steady drumbeat of advocacy about the urgency of completing eradication and delivering a polio-free world, other voices from within the world of public health and without, have raised fundamental questions about the wisdom of focusing so much attention on a single disease without paying equal attention to strengthening health systems in general and, in particular, whether polio eradication is feasible in contexts where health systems are unable to deliver routine immunisations for other vaccine preventable childhood diseases.

\section{Cat and mouse game}

Transmission of polio can be interrupted through intense vaccine campaigns based around national and subnational immunisation days and supplementary immunisation campaigns, but to stay polio free requires a robust routine immunisation programme. As Yemane Berhane and colleagues have pointed in the context of Nigeria, while campaign style immunisation might be sufficient to temporarily stop polio transmission, "without a functioning immunisation system, Nigeria will remain at risk for renewed transmission and will continue to pose a risk to other countries." 1

The absence of robust routine childhood immunisation systems makes countries where polio has been stamped out vulnerable if the disease is imported, as has happened in countries surrounding Nigeria. Thus in 2001, Chad was being congratulated for ending polio transmission. But because routine immunisation systems were weak, cases coming in from across the border were able to re-establish the disease, and at the end of 2011 Chad had the largest number of cases per million population of any country in the world. Similarly, Angola had been polio free from 2001 till 2007, when an imported case led to an outbreak in the capital Luanda which spread to other districts in the country, as well as to the Democratic Republic of Congo, where it is now entrenched.

This cat and mouse game of intense campaigns in countries without robust immunisation systems is not a sustainable long term strategy and was not part of the original vision for polio eradication. The 1988 World Health Assembly resolution calling for eradication by 2000 regarded the polio campaign as one component of the Expanded Programme of Immunisation (EPI), which was launched in 1974 and had the goal of universal immunisation of children by 1990 for diphtheria, tetanus, pertussis, measles, polio, and tuberculosis. This immunisation programme was part of WHO's vision of achieving "health for all" by 2000 . The resolution specifically mentioned that polio eradication was to be carried out in ways which strengthened the EPI, health infrastructure, and primary healthcare. This was in keeping with WHO's global health philosophy of the time, which saw overall strengthening of health systems as the main way to achieve better health outcomes, as opposed to individual disease eradication programmes.

But as the deadlines slipped by and funding became more uncertain, the polio campaign took on a life and rationale of its own. While this helped advocacy and fund raising, it has had other consequences as well.

The consequences of the separation between the polio programme and routine childhood immunisation is perhaps best illustrated by the fact that the countries where routine immunisation for other childhood diseases are the weakest, tend to be the countries where polio persists. If we use the extent of DTP3 (three doses of diphtheria, tetanus, and pertussis) immunisation as a measure of routine childhood immunisation in a country, we find a relation between low childhood immunisation rates and the persistence of polio. WHO estimates that in 2010 , roughly 19 million children in the world had not received DTP3 in routine immunisation programmes during their first year of life. ${ }^{2}$ Half of these children lived in Nigeria, India, and the Democratic Republic of Congo (DRC). Nigeria and the Democratic Republic of Congo have persistent poliovirus transmission, and India has only recently managed to stamp out polio, after great difficulty. The two other countries where polio persists, Pakistan and Afghanistan, are among the 10 countries that contain the bulk of children who have not had routine DTP immunisation.

The weakness of routine immunisation services both in the countries where polio persists, and in countries that are vulnerable to fresh outbreaks, makes eradication difficult but still possible. The $99 \%$ decrease in polio cases since the eradication campaign was launched demonstrates this. But once wild polioviruses are eradicated, and eradication is certified, another complication will have to be dealt with.

\section{New risks}

One of the paradoxes of the polio eradication programme is that even after wild polioviruses are eradicated, cases of poliomyelitis caused by the viruses derived from the oral polio vaccine are likely to continue for some years. The attenuated Sabin strain virus used in the oral polio vaccine can revert to a virulent state, and it transmits as easily and causes disease as severe as the wild poliovirus. In 2011, there were 58 cases of disease caused by a circulating vaccine derived virus. Of these, 34 cases were in Nigeria, from a chain of transmission that began in $2005 .^{3}$

These 58 cases could be viewed as an acceptable risk compared with the 650 cases caused by the wild poliovirus in 2011 globally. But as the number of polio cases caused by wild poliovirus reduces, the relative risk posed by vaccine derived viruses and vaccine associated paralytic polio will rise. To reduce this risk, the eradication initiative has suggested that use of the oral vaccine should stop globally around five years after certification is achieved. But population immunity will still need to be maintained against residual circulating vaccine derived viruses. This will require either monovalent live vaccines or injectable inactivated vaccines. There is no indication of how long immunisation will have to be continued after the eradication of wild polioviruses, or how much it will cost.

Though the 2012 target for eradicating wild poliovirus still stands, nobody can predict whether it will be met. But it is clear that eradication will be a long drawn out process that is more complex than indicated by the simplistic advocacy messages of the eradication initiative and will cost more than the $\$ 2.13 \mathrm{bn}$ that it has budgeted for till the end of 2013. Nevertheless, it remains an important public health goal.

Competing interests: The author has completed the ICJME unified disclosure form at www.icmje.org/coi_disclosure.pdf (available on request from the corresponding author) and declares no support from 
any organisation for the submitted work; no financial relationships with any organisation that might have an interest in the submitted work in the previous three years; TA was employed by WHO during 2009 as a communications officer. He has also been a temporary adviser for WHO communication workshops and meetings.

Provenance and peer review: Commissioned; externally peer reviewed.
1 Berhane Y, Clements CJ, Ndiaye JM, Taylor P. Has routine immunisation in Africa become endangered? Lancet Infect Dis 2009;9:655-6.

2 Global routine vaccine coverage 2010. WHO Wkly Epidemiol Rec 2011;46:510-1.

Global Polio Eradication Initiative. Circulating vaccine-derived poliovirus (cVDPV) 2000-2012. www.polioeradication.org/Dataandmonitoring/Poliothisweek/ Circulatingvaccinederivedpoliovirus.aspx.

Cite this as: BMJ 2012;344:e2398

(c) BMJ Publishing Group Ltd 2012 


\section{Figures}

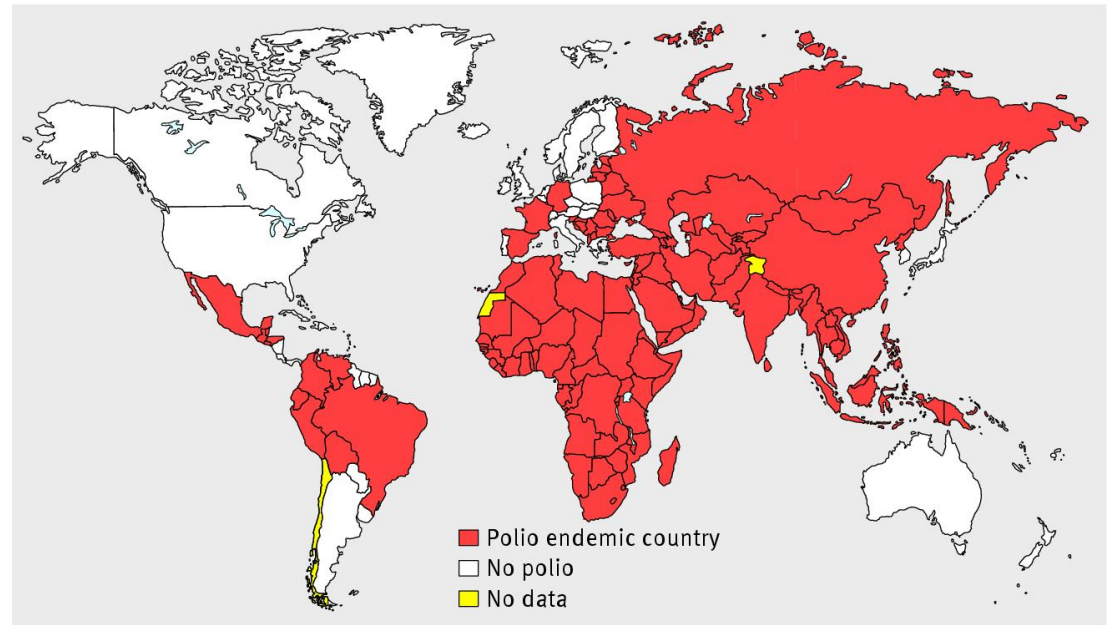

Countries affected by polio, 1988

[Image: WHO]

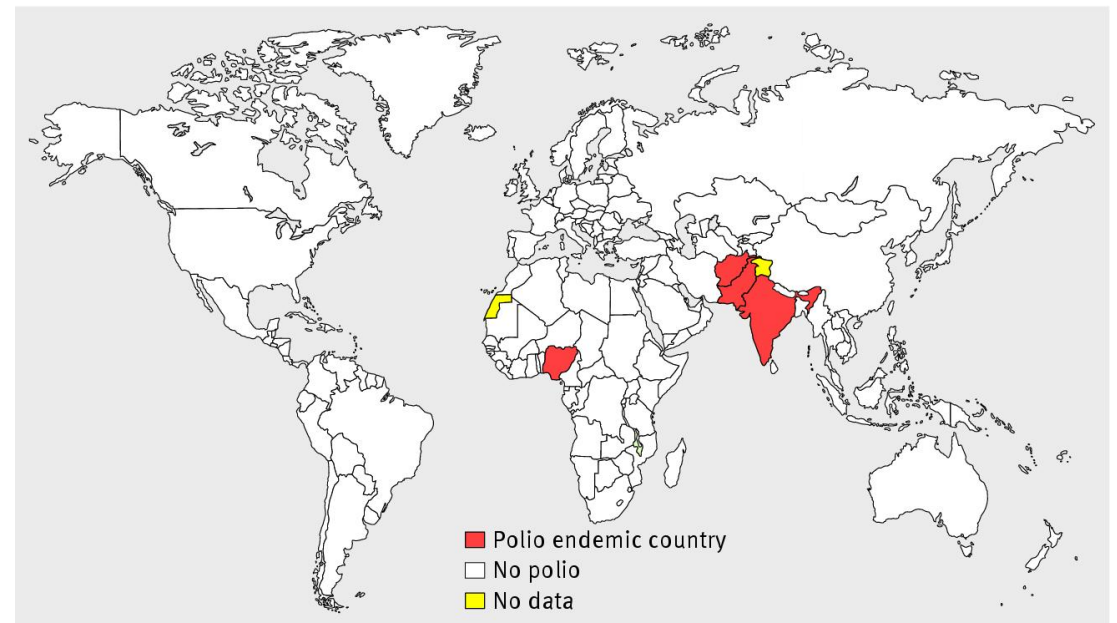

Countries affected by polio, 2010

[Image: $W H O$ ] 